\title{
Solifenacin may improve sleep quality in patients with overactive bladder and sleep disturbance
} Takao T, Tsujimura A, Yamamoto K, Fukuhara S, Nakayama J, Matsuoka Y, Miyagawa Y, Nonomura N Department of Urology, Osaka University Graduate School of Medicine, Suita, Japan Urology. 2011; 78: 648-52

Objective: To examine the effect of solifenacin for not only overactive bladder symptoms but also sleep disturbance. Nocturia and urgency are independent factors for sleep disturbance.

Methods: Fifteen male patients with overactive bladder symptoms and sleep disturbance were enrolled in this study. The overactive bladder symptoms score (OABSS) and Athens insomnia scale (AIS) were used as a subjective questionnaire for overactive bladder symptoms and insomnia. The Actiwatch-16 (Mini-MitterRespironics, Inc., Bend, OR) was used as an objective measurement tool for insomnia. Total sleep time, sleep efficiency, sleep latency, wake-after-sleep onset, and number of awakenings were measured by the Actiwatch. We evaluated the changes of each parameter before and 8 weeks after the administration of solifenacin. Statistical comparisons before and after the administration were made using the Wilcoxon signed-rank test. To examine the relation between OABSS and AIS, Spearman's testing was used for correlations between independent variables and $\mathrm{P}<0.05$ was considered statistically significant.

Results: Total OABSS and total AIS were significantly improved after administration of solifenacin. The cat- 


\section{Urological Survey}

egories of urgency and nocturia in OABSS and the categories of awakening during the night and sleep quality in AIS were also significantly improved. The Actiwatch study showed that total sleep time and sleep efficiency were significantly improved. The decrease of AIS was significantly correlated with the decrease of urgency ( $\rho$ $=0.635, \mathrm{P}=0.0175$ ) but not with nocturia.

Conclusion: The treatment of urgency by solifenacin may improve not only overactive bladder symptoms but also sleep disturbance.

\section{Editorial Comment}

In this interesting report by Takao et al. they objectively assess the impact of solifenacin on sleep disturbance in male patients suffering from OAB symptoms. They used an electronic simple device (Actiwatch) for 1 week before and after treatment and validated questionnaires to assess the effects of a daily drug use of $5 \mathrm{mg}$ for 8 weeks.

Results confirmed an improvement in urgency and nocturia. A significant decrease in awakenings during the night and an improvement in quality of sleep were also detected.

Although multifactorial sleep disturbance can be at least partially deteriorated by nocturnal frequency, and therefore improving bladder capacity, decreasing afferent sensibility and night time urine overproduction are targets to be aimed at. In order to obtain more consistent data, prospective randomized placebo controlled studies and head-to-head comparison with other antimuscarinic agents are warranted.

Dr. Ricardo Miyaoka

State University Campinas

Campinas, SP, Brazil

E-mail:rmiyaoka@uol.com.br 